\title{
Theoretical relation between halo current-plasma energy displacement/deformation in EAST
}

\author{
Shahab Ud-Din Khan ${ }^{1} \cdot$ Salah Ud-Din $\mathrm{Khan}^{2} \cdot$ Yuntao Song ${ }^{3} \cdot$ Chen Dalong $^{3}$
}

Received: 20 November 2017 / Accepted: 25 February 2018/Published online: 28 April 2018

(C) The Author(s) 2018

\begin{abstract}
In this paper, theoretical model for calculating halo current has been developed. This work attained novelty as no theoretical calculations for halo current has been reported so far. This is the first time to use theoretical approach. The research started by calculating points for plasma energy in terms of poloidal and toroidal magnetic field orientations. While calculating these points, it was extended to calculate halo current and to developed theoretical model. Two cases were considered for analyzing the plasma energy when flows down/upward to the diverter. Poloidal as well as toroidal movement of plasma energy was investigated and mathematical formulations were designed as well. Two conducting points with respect to $(R, Z)$ were calculated for halo current calculations and derivations. However, at first, halo current was established on the outer plate in clockwise direction. The maximum generation of halo current was estimated to be about 0.4 times of the plasma current. A Matlab program has been developed to calculate halo current and plasma energy calculation points. The main objective of the research was to establish theoretical relation with experimental results so as to precautionary evaluate the plasma behavior in any Tokamak.
\end{abstract}

Keywords Plasma energy $\cdot$ Magnetic field points $\cdot$ Horizontal/vertical forces $\cdot$ Halo current $\cdot$ Mathematical model

\section{List of symbols}

$A\left(\mathrm{~m}^{2}\right)$

$a^{\prime}(\mathrm{m})$

$a(\mathrm{~m})$

$A_{\mathrm{p}}$

$A$

$B_{0}(\mathrm{~T})$

$B_{x}(\mathrm{~T})$
Plasma area

Minor radius

Radius of current loop

Median flow path length

Indicates the Plasma tilt positions

Initial magnetic field

Magnetic field in $x$-direction

Electronic supplementary material The online version of this article (https://doi.org/10.1007/s40094-018-0276-1) contains supplementary material, which is available to authorized users.

Shahab Ud-Din Khan

shahab.furqan@gmail.com

Salah Ud-Din Khan

drskhan@ksu.edu.sa

1 National Tokamak Fusion Program, Pakistan Atomic Energy Commission (PAEC), Islamabad 3329, Pakistan

2 Sustainable Energy Technologies Center, King Saud University, P.O.Box 800, Riyadh 11421, Kingdom of Saudi Arabia

3 Institute of Plasma Physics, Chinese Academy of Sciences, P.O.Box 1126, Hefei 230031, Anhui, People's Republic of China

$B_{z}(\mathrm{~T}) \quad$ Magnetic field in $z$-direction

$B_{\theta}(\mathrm{T}) \quad$ Poloidal magnetic field

$B$ (T) Magnetic field

$b$ (m) $\quad z$-axis coordinate of current loop

$B_{\text {tor }}(\mathrm{T}) \quad$ Toroidal magnetic field

$E(\mathrm{k}) \quad$ Second type of elliptic integral

$I_{\mathrm{p}}$ (MA) Total plasma toroidal current

$K(\mathrm{~m}) \quad$ Elongation

$K(\mathrm{k}) \quad$ First type of elliptic integral

$R^{\prime}(\mathrm{m}) \quad$ Major radius

$r_{\mathrm{p}}(\mathrm{m}) \quad$ Plasma radial length

$\delta r_{\mathrm{p}} \quad$ Radial kink mode amplitude

$Z \quad$ Z-axis coordinate of calculated point

$\Delta \quad$ Triangularity

$\phi \quad$ Magnetic flux

$\delta z_{\mathrm{p}} \quad$ Vertical kink mode amplitude

$z_{\mathrm{p}}(\mathrm{m}) \quad$ Plasma vertical length

\section{Introduction}

During the disruption scenario in plasma, eddy and halo currents were considered to be the most important sources that came out as a result of this phenomenon [1]. The 
components which affect primarily are diverter, First Wal (FW) and other main components, because plasma is unstable in vertical displacement. In this scenario, plasma moved upward and downward resulting into plasma disruption, and as a result, halo current has been generated helically. This halo current was produced in SOL component and flows into the vacuum vessel through in-vessel components that may give rise to large force acting on the vessel and in-vessel components [2]. The number of research articles has been published to investigate the halo current in other Tokamak devices such as JET [3], JT-60U [4], and NSTX [5]. Like in Experimental Advanced Superconducting Tokamak (EAST) reactor [6-8], there are some failures of the feedback control in VD event caused by disruption. The EAST reactor is designed by Institute of Plasma Physics, Chinese Academy of Sciences, P.R. China which is considered to be the best superconducting and advanced Tokamak in the world.

Recently, EAST has been updated and had achieved longer pulse generations at high current mode. Main parameters of EAST reactor are given in Table 1 [9].

The EAST has been demonstrated as long-pulsed plasma operations with toroidal field $B_{\mathrm{t}} \leq 3.5 \mathrm{~T}$ and plasma current $I_{\mathrm{p}} \leq 1 \mathrm{MA}$ [10-12]. The EAST upper diverter has been upgraded with $\mathrm{W} / \mathrm{Cu}$ plasma facing components (PFCs) with ITER like W-monoblack [13]. The lower diverter has not been upgraded using graphite tiles for the first wall. It has a central dome, but the upper diverter does not equip with it. In addition, an EAST disruption database has been built [14] and is useful for quickly selecting disruptive discharges and their relevant parameters. Nearly, $27 \%$ of discharges terminated in a disruption [15].

In this reactor, some sensors have been installed for calculating the halo currents at different locations, as given in Table 2. In these experiments, it was observed that the halo current first spread out on outer baffle plate

Table 1 Parameters of EAST [9]

\begin{tabular}{ll}
\hline Parameters & Values \\
\hline Major radius & $1.7 \mathrm{~m}$ \\
Minor radius & $0.4 \mathrm{~m}$ \\
Triangularity & $0.6-0.8$ \\
Pulse length & $1000 \mathrm{~s}$ \\
Plasma current & $1 \mathrm{MA}$ \\
Torroidal field & $3.5 \mathrm{~T}$ \\
H\&CD & $\sim 25 \mathrm{MW}$ \\
Configuration & Double-null diverter \& single null diverter \\
Elongation(K) & $1.6-2$ \\
Aspect ratio(R/a) & 4.25 \\
\hline
\end{tabular}

then moves to dome and finally return back to plasma. At first, we have developed model (2) to calculate magnetic field produced by circular current loop. Furthermore, we considered two cases to analyze the plasma when it flows up/down direction as observed by model (2) calculation data. Second, we have theoretically calculated horizontal/vertical forces connected with model (2). It was observed that generation of halo current was huge and it has strong field at the middle and weak or cancelling effects appear at the sideways positions. Third, during disruption, magnetic field appears at the conducting points consisting of large number of magnetic flux. Therefore, halo current model (18) has been developed using Eqs. (9 and 15) with specific parameters $\left(r_{\mathrm{p}}, z, z_{\mathrm{p}}\right.$, $\left.u_{\text {shift }}, \phi\right)$. A Matlab program has been developed to calculate halo current and magnetic field calculation points. The achievable maximum halo current was about 0.4 times of the plasma current and its maximum TPF values was 0.65 as estimated by set of sensors. EAST halo current is $10 \mathrm{KA}$ for one cassette and total estimation is 400 KA by model (18).

Some of the work that has already been published for halo current is given as under

- Strauss with his team carried out heavy simulation using M3D code for calculating the halo current and plasma disruption conditions [16].

- Long et al. experimentally calculated the values of eddy and halo current in EAST reactor while conducting set of VDE experiments [17].

- Other impressive contributions by Strauss and his team for assessing and simulation of halo current in ITER through VDE instabilities and developed 3D models for plasma disruption conditions [18].

- Fantechi and Crutzen modeled the plasma FW contact under vertical instabilities with a 3D eddy current simulation. The simulation worked for transferring the electric current (halo current) to $\mathrm{FW}$ component through VDE [19].

\section{Model of magnetic field calculation points for Tokamak}

In this research work, a new model for calculating different aspects of Tokamak reactor has been designed. In the recent past, number of work has been published already about the shaping and geometrical description of plasma $[20,21]$. One of the important problem is to identify and simulate the plasma shape and control including the numerical calculations on elongated and shaped equilibria [22]. In this paper, a new model was developed on the basis of an applied mathematical approach [23], that is 
Table 2 EAST halo diagnostics [17]

\begin{tabular}{llll}
\hline Position & Poloidal & Toroidal & Function \\
\hline Center column & (upper/middle/lower) & - & Measure poloidal halo current \\
Upper diverter & 10 & 4 & Measure halo current through mono block and water cooling tubes \\
Lower diverter & 8 (plate support leg) & 4 & Halo current in diverter support \\
& 6 (copper plate of dome) & 2 & Directly measure halo current in dome \\
\hline
\end{tabular}

$R^{\prime}=R_{0}+a_{\mathrm{p}} \cos (\theta+\delta \sin \theta)$

$Z^{\prime}=Z_{0}+k a_{\mathrm{p}} \sin \theta$

where " $k$ " is elongation, " $\delta$ " is triangularity, " $a$ " is minor radius, and " $R_{0}$ " is the major radius. Using the algebraic techniques, as the magnetic field is $B=B_{X}+B_{Y}$, we obtained $B_{x}$ and $B_{z}$ using shape model (1) in energy integral [24]. The calculated points are given as under

$B_{x}=B_{r} \cos \alpha-B_{\theta} \cos \left(\frac{\pi}{2}-\alpha\right)=B_{r} \cos \alpha-B_{\theta} \sin \alpha$

$B_{z}=B_{r} \sin \alpha+B_{\theta} \sin \left(\frac{\pi}{2}-\alpha\right)=B_{r} \sin \alpha+B_{\theta} \cos \alpha$.

Magnetic field produced by a circular current loop [25] is

$$
\begin{aligned}
B_{r}= & \frac{\mu_{0} I}{2 \pi r} \frac{z-b}{\left[(a+r)^{2}+(z-b)^{2}\right]^{\frac{1}{2}}} \\
& {\left[-K(k)+\frac{a^{2}+r^{2}+(z-b)^{2}}{(a-r)^{2}+(z-b)^{2}} E(K)\right] } \\
B_{\theta}= & \left.\frac{\mu_{0} I}{4 \pi r} \frac{1}{\left.[a+r)^{2}+(z-b)^{2}\right]^{\frac{1}{2}}}\right] \\
& {\left[K(k)+\frac{a^{2}-r^{2}-(z-b)^{2}}{(a-r)^{2}+(z-b)^{2}} E(K)\right] } \\
K(k)= & \int_{0}^{\pi / 2} \frac{\mathrm{d} \alpha}{\sqrt{1-K^{2} \sin ^{2}} \alpha}, E(k)=\int_{0}^{\pi / 2} \sqrt{1-K^{2} \sin ^{2} \alpha \mathrm{d} \alpha,}
\end{aligned}
$$

where $R_{0}=1.7-1.8, \quad B_{0}=3.5 \mathrm{~T}, \quad$ plasma current $I_{\mathrm{p}} \leq 1 \mathrm{MA}$.

\section{Plasma orientation and development of halo current}

In case of plasma, it was observed that some balancing and unbalancing forces acted upon, like the case of plasma as torus which is very close to outboard wall. At this stage, poloidal field has different radial locations and have variable sideways forces as well. In this case, one side of torus has higher values than other side and poloidal field has different values at different locations.

Case 1 In this case, we considered the plasma at a position $(R, Z)$ from the origin and assumed that during the disruption occurs (Fig. 1), the plasma moves horizontally [26, 27]. The governing equations are given as under

$F_{\mathrm{h}}=F_{1} \cos \alpha+F_{1} \sin \alpha, \alpha=0,180,360 \ldots$

$F_{\mathrm{h}}=F_{1} \cos \alpha$.

Since

$F_{\mathrm{h}}=B I \Delta x \cos \alpha$,

where

$\Delta x=\cos \alpha \delta x_{\mathrm{p}}$.

Therefore

$F_{\mathrm{h}}=B I_{\mathrm{p}}\left(\cos \alpha \delta x_{\mathrm{p}}\right) \cos \alpha$.

Since the plasma is in the range of $0 \rightarrow 2 \pi$, therefore

$F_{\mathrm{h}}=\int_{0}^{2 \pi} B I_{\mathrm{p}} \cos ^{2} \alpha \delta x_{\mathrm{p}} \mathrm{d} \alpha$.

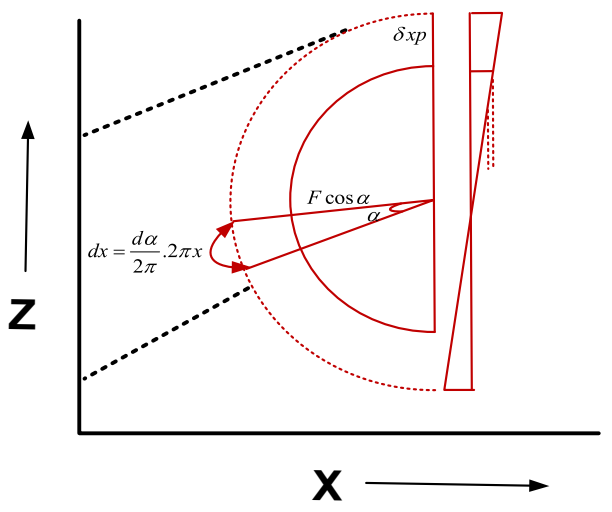

Fig. 1 Plasma orientation (case 1) 
$F_{\mathrm{h}}=B I_{\mathrm{p}} \delta x_{\mathrm{p}} \int_{0}^{2 \pi} \cos ^{2} \alpha \mathrm{d} \alpha=\pi B I_{\mathrm{p}} \delta x_{\mathrm{p}}$.

Substitution of Eqs. (2) into (9) gives the changes in magnetic field while applying the horizontal forces on the plasma giving plasma position in different points, as presented in Table 3. For static position, each cross section depends on $\mathrm{a}, \mathrm{b}, \mathrm{r}$, and $\mathrm{z}$. For one cross section, the peak values of $r$ and $z$ are $( \pm 5.2, \pm 4.37)$; therefore, $\left(B_{\mathrm{r}(\max , \text { up }) \text {, }}\right.$ $\left.B_{\mathrm{r}(\max , \text { down })}\right)=(0.3918,-0.3847)$. Then, the current loop of magnetic field range was $\left(B_{x}, B_{z}\right)_{\max }=(0.3862,0.1698)$ for selected degree of cos (alpha) and plasma one crosssectional area energy was calculated to be $8.0262 \mathrm{e}+004$. For tilting position, peak values of $r$ and $z$ were $( \pm 5.4, \pm 4.17) ; \quad$ therefore,$\quad\left(B_{\mathrm{r}(\max , \text { up })}, \quad B_{\mathrm{r}(\max , \text { down })}\right)-$ $=(0.4225,-0.4247)$. Hence, the current loop of magnetic field range was $\left(B_{x}, B_{z}\right)_{\max }=(0.4221,0.1974)$ for selected degree of $\cos (\mathrm{alpha})$ and calculated plasma one cross-sectional area energy was $8.0258 \mathrm{e}+004$ (see Table 3).

Case 2 In this case, we considered plasma at a position $(R, Z)$ from the origin and assumed that during disruption occurs (Fig. 2), plasma moves vertically downward $[26,27]$. The governing equations are given as under

$F_{v}=F_{2} \cos \beta+F_{2} \sin \beta, \beta=90,270 \ldots$

Then

$F_{v}=F_{2} \sin \beta$

Since

$F_{v}=B I \Delta z \sin \beta$,

Where

$\Delta z=-\sin \beta \delta z_{\mathrm{p}}$

Therefore

$F_{v}=B I_{\mathrm{p}}\left(-\sin \beta \delta z_{\mathrm{p}}\right) \sin \beta$.

Since the plasma is in the range of $0 \rightarrow 2 \pi$ :

$F_{v}=-\int_{0}^{2 \pi} B I_{\mathrm{p}} \sin ^{2} \beta \delta z_{\mathrm{p}} \mathrm{d} \beta$.

and

$F_{v}=-B I_{\mathrm{p}} \delta z_{\mathrm{p}} \int_{0}^{2 \pi} \sin ^{2} \beta \mathrm{d} \beta=-\pi B I_{\mathrm{p}} \delta z_{\mathrm{p}}$.

Changes in magnetic field can be described by substituting Eq. (2) into Eq. (15) to get the vertical forces applies to the plasma so as to calculates the plasma position at different points. Like in Case-1, each cross section depended upon $a, b, r$, and $z$. For one cross section, the peak values of $r, z$ were $( \pm 3.5, \pm 2.3)$; therefore, $\left(B_{\mathrm{r}(-}\right.$ max,up $\left.), B_{\mathrm{r}(\text { max,down })}\right)=(0.1865,-0.4652)$. Then, the current loop of magnetic field range was $\left(B_{x}, B_{z}\right)_{\max }=(0.4637$, $0.1995)$ for selected degree of $\cos ($ alpha) and plasma one cross-sectional area energy was $8.0457 \mathrm{e}+004$. For tilting position, peak values of $r$ and $z$ were $( \pm 3.8, \pm 2.5)$; therefore, $\left(B_{\mathrm{r}(\max , \text { up }),} B_{\mathrm{r}(\max , \text { down })}\right)=(0.2762,-0.3851)$. The current loop of magnetic field range was $\left(B_{x}, B_{z}\right)_{\max }$ $=(0.5747,0.2525)$ for selected degree of $\cos ($ alpha $)$ and the calculated values of plasma one cross-sectional area energy was $8.0484 \mathrm{e}+004$ (see Table 4).

During VDE, plasma changes the area under vertical and horizontal forces and the magnetic field changes in radial direction at center of plasma with major radius $(R)$, $x-z$ displacement and plasma radial/vertical length $\left(z_{\mathrm{p}}, r_{\mathrm{p}}\right)$. During this plasma change, magnetic field of each point can be calculated by model (2) (vertical and horizontal). The magnetic field appears at the conducting points consisting of large number of magnetic flux, which are given below as

$$
\begin{aligned}
\phi & =\phi_{1}+\phi_{2}+\phi_{3}+\phi_{4} \cdots+\phi_{n} \\
& =B A_{1} \cos \phi_{1}+B A_{2} \cos \phi_{2}+B A_{3} \cos \phi_{3} \cdots B A_{n} \cos \phi_{n} \\
& =B\left(A_{1} \cos \phi_{1}+A_{2} \cos \phi_{2}+A_{3} \cos \phi_{3} \cdots A_{n} \cos \phi_{n}\right) \\
\phi_{\text {total }} & =B \sum_{i=1}^{n} A_{i} \cos \phi_{i} .
\end{aligned}
$$

At the conducting points, generation of halo current was huge and it has strong field at the middle and weak forces or cancelling effects appeared at the sideways positions. Due to horizontal and vertical forces, some balancing and unbalancing forces appeared and it was expected that asymmetric plasma positions were along the toroidal coordinates. The poloidal halo current which balances the plasma vertical displacement was toroidally asymmetric. According to the plasma positions, these halo currents have different flow poloidal paths along with the toroidal coordinates. Similarly, due to the asymmetry of poloidal halo currents, the second sideways force occured and the sum of these forces gave sideways balancing forces as well:

$u(\alpha, \beta)=c \delta x_{\mathrm{p}} \cdot \cos \alpha+d \delta z_{\mathrm{p}} \cdot \sin \beta$.

The total forces includes horizontal and vertical and the summation of these forces acted upon the plasma VDE direction gives halo current as given below:

$I_{\text {halo }}=\frac{2}{B}\left[\frac{F_{\mathrm{h}}}{\left(A_{\mathrm{p}}-z_{\mathrm{p}}\right) u_{1}}+\frac{F_{v}}{\left(A_{\mathrm{p}}-x_{\mathrm{p}}\right) u_{2}}\right]+\phi_{\text {total }}$.

During the course of disruption, halo and eddy currents considered to be the main source of electro-mechanical 
Table 3 Magnetic field with horizontal displacement by Eqs. (2 and 9)

\begin{tabular}{|c|c|c|c|c|c|c|c|c|}
\hline No. & $B_{x}$ & $B_{z}$ & No. & $B_{x}$ & $B_{z}$ & No. & $B_{x}$ & $B_{z}$ \\
\hline 1 & $\begin{array}{r}-0.0277 \\
-0.0046\end{array}$ & $0.3162,0.0523$ & 66 & $\begin{array}{c}0.2130,- \\
0.2098\end{array}$ & $-0.0053,-0.0421$ & 132 & $\begin{array}{c}0.2004,- \\
0.2151\end{array}$ & $0.0998,0.0615$ \\
\hline 3 & $-0.0030-0.0017$ & $0.0347,0.0193$ & 68 & $\begin{array}{c}0.1842,- \\
0.1804\end{array}$ & $-0.0140,-0.0456$ & 134 & $\begin{array}{c}0.2353,- \\
0.2441\end{array}$ & $0.0757,0.0327$ \\
\hline 5 & $-0.0008,0.0005$ & $0.0060,-0.0055$ & 70 & $\begin{array}{c}0.1390,- \\
0.1350\end{array}$ & $-0.0218,-0.0453$ & 136 & $\begin{array}{l}0.2623,- \\
0.2665\end{array}$ & $0.0554,0.0086$ \\
\hline 7 & $0.0013,0.0020$ & $-0.0151,-0.0228$ & 72 & $\begin{array}{c}0.0930,- \\
0.0889\end{array}$ & $-0.0272,-0.0425$ & 138 & $\begin{array}{c}0.2810,- \\
0.2820\end{array}$ & $0.0378,-0.0117$ \\
\hline 9 & $0.0025,0.0020$ & $-0.0284,-0.0228$ & 74 & $\begin{array}{c}0.0692,- \\
0.0645\end{array}$ & $-0.0274,-0.0389$ & 140 & $\begin{array}{c}0.2909,- \\
0.2902\end{array}$ & $0.0223,-0.0286$ \\
\hline 11 & $0.0013,0.0020$ & $-0.0151,-0.0228$ & 76 & $\begin{array}{c}0.0042,- \\
0.0409\end{array}$ & $0.2000,0.1924$ & 142 & $\begin{array}{c}0.2903,- \\
0.2891\end{array}$ & $0.0083,-0.0422$ \\
\hline 13 & $0.0025,0.0028$ & $-0.0284,-0.0317$ & 78 & $0.0261-0.0572$ & $0.1698,0.1598$ & 144 & $\begin{array}{c}0.2784,- \\
0.2770\end{array}$ & $\begin{array}{c}-0.0042,- \\
0.0524\end{array}$ \\
\hline 15 & 0.0029 & -0.0326 & 80 & $\begin{array}{c}0.0692,- \\
0.0919\end{array}$ & $0.1279,0.1122$ & 146 & $\begin{array}{c}0.2454,- \\
0.2430\end{array}$ & $\begin{array}{c}-0.0162,- \\
0.0581\end{array}$ \\
\hline 16 & $0.0210,-0.0674$ & $0.2698,0.2680$ & 82 & $\begin{array}{c}0.1110,- \\
0.1277\end{array}$ & $0.0990,0.0773$ & 148 & $\begin{array}{c}0.2998,- \\
0.2983\end{array}$ & $\begin{array}{c}-0.0558,- \\
0.0526\end{array}$ \\
\hline 18 & $-0.0385,-0.0367$ & $0.1961,0.1945$ & 84 & $\begin{array}{c}0.1504,- \\
0.1618\end{array}$ & $0.0769,0.0492$ & 150 & $\begin{array}{c}0.1986,- \\
0.2171\end{array}$ & $0.1501,0.1073$ \\
\hline 20 & $0.0087,-0.0300$ & $0.1398,0.1383$ & 86 & $\begin{array}{c}0.1851,- \\
0.1918\end{array}$ & $0.0587,0.0256$ & 152 & $\begin{array}{c}0.2340,- \\
0.2513\end{array}$ & $0.1147,0.0685$ \\
\hline 22 & $0.0464,-0.0652$ & $0.1062,0.1059$ & 88 & $\begin{array}{c}0.2131,- \\
0.2161\end{array}$ & $0.0430,0.0055$ & 154 & $\begin{array}{c}0.2668,- \\
0.2803\end{array}$ & $0.0866,0.0366$ \\
\hline 24 & $0.0872,-0.1010$ & $0.0830,0.0830$ & 90 & $\begin{array}{c}0.2328,- \\
0.2334\end{array}$ & $0.0293,-0.0115$ & 156 & $\begin{array}{c}0.0336,- \\
0.3013\end{array}$ & $0.0632,0.0100$ \\
\hline 26 & $0.1257,-0.1353$ & $0.0649,0.0649$ & 92 & $\begin{array}{c}0.2431,- \\
0.2420\end{array}$ & $0.0169,-0.0256$ & 158 & $\begin{array}{c}0.3143,- \\
0.3156\end{array}$ & $0.0433,-0.0125$ \\
\hline 28 & $0.1599,-0.1660$ & $0.0497,0.0498$ & 94 & $\begin{array}{c}0.2421,- \\
0.2401\end{array}$ & $0.0054,-0.0366$ & 160 & $\begin{array}{c}0.3242,- \\
0.3237\end{array}$ & $0.0257,-0.0313$ \\
\hline 30 & $0.1879,-0.1910$ & $0.0365,0.0365$ & 96 & $\begin{array}{c}0.2290,- \\
0.2243\end{array}$ & $-0.0162,-0.0553$ & 162 & $\begin{array}{c}0.3249,- \\
0.3246\end{array}$ & $0.0100,-0.0468$ \\
\hline 32 & $0.2081,-0.2089$ & $0.0245,0.0246$ & 98 & $\begin{array}{c}0.2140,- \\
0.1820\end{array}$ & $-0.1809,-0.2119$ & 164 & $\begin{array}{c}0.2914,- \\
0.2890\end{array}$ & $\begin{array}{c}-0.0062,- \\
0.0548\end{array}$ \\
\hline 34 & $0.2186,-0.2175$ & $0.0137,0.0137$ & 100 & $\begin{array}{c}0.1653,- \\
0.1391\end{array}$ & $-0.1531,-0.1770$ & 166 & $\begin{array}{c}0.3086,- \\
0.3078\end{array}$ & $\begin{array}{c}-0.0030,- \\
0.0564\end{array}$ \\
\hline 36 & $0.2179,-0.2153$ & $0.0037,0.0038$ & 102 & $\begin{array}{c}0.0487,- \\
0.0806\end{array}$ & $0.1958,0.1798$ & 168 & $\begin{array}{c}0.2806,- \\
0.2920\end{array}$ & $0.1320,0.0757$ \\
\hline 38 & $0.2042,-0.2006$ & $-0.0054,-0.0053$ & 104 & $\begin{array}{c}0.0907,- \\
0.1153\end{array}$ & $0.1464,0.1253$ & 170 & $\begin{array}{c}0.3125,- \\
0.3218\end{array}$ & $0.0994,0.0403$ \\
\hline 40 & $0.1761,-0.1717$ & $-0.0136,-0.0135$ & 106 & $\begin{array}{c}0.1326,- \\
0.1512\end{array}$ & $0.1127,0.0860$ & 172 & $\begin{array}{c}0.3380,- \\
0.3435\end{array}$ & $0.0724,0.0107$ \\
\hline 42 & $0.1321,-0.1274$ & $-0.0206,-0.0215$ & 108 & $\begin{array}{c}0.1722,- \\
0.1851\end{array}$ & $0.0870,0.0549$ & 174 & $\begin{array}{c}0.3570,- \\
0.3583\end{array}$ & $0.0494,-0.0143$ \\
\hline 44 & $0.0851,-0.0804$ & $-0.0261,-0.0257$ & 110 & $\begin{array}{c}0.1574,- \\
0.1548\end{array}$ & $0.0663,0.0290$ & 176 & $\begin{array}{c}0.3664,- \\
0.3668\end{array}$ & $0.0293,-0.0354$ \\
\hline 46 & $-0.0067,-0.0298$ & $0.2131,0.2102$ & 112 & $\begin{array}{c}0.2071,- \\
0.2146\end{array}$ & $0.0486,0.0071$ & 178 & $\begin{array}{c}0.3662,- \\
0.3666\end{array}$ & $0.0126,-0.0515$ \\
\hline 48 & $0.0121,-0.0400$ & $0.1510,0.1449$ & 114 & $\begin{array}{c}0.2348,- \\
0.2381\end{array}$ & $0.0332,-0.0114$ & 180 & $\begin{array}{c}0.3363,- \\
0.3362\end{array}$ & $0.0102,-0.0486$ \\
\hline 50 & $0.0545,-0.0753$ & $0.1144,0.1023$ & 116 & $\begin{array}{c}0.2541,- \\
0.2547\end{array}$ & $0.0194,-0.0267$ & 182 & $\begin{array}{c}0.3424,- \\
0.3464\end{array}$ & $0.1074,0.0426$ \\
\hline 52 & $0.0959,-0.1111$ & $0.0890,0.0706$ & 118 & $\begin{array}{c}0.2641,- \\
0.2632\end{array}$ & $0.0069,-0.0390$ & 184 & $\begin{array}{c}0.3862,- \\
0.3871\end{array}$ & $0.0816,0.0109$ \\
\hline
\end{tabular}


Table 3 (continued)

\begin{tabular}{|c|c|c|c|c|c|c|c|c|}
\hline No. & $B_{x}$ & $B_{z}$ & No. & $B_{x}$ & $B_{z}$ & No. & $B_{x}$ & $B_{z}$ \\
\hline 54 & $0.1349,-0.1453$ & $0.0694,0.0448$ & 120 & $\begin{array}{c}0.2635,- \\
0.2618\end{array}$ & $-0.0047,-0.0477$ & 186 & $\begin{array}{c}0.3782,- \\
0.3814\end{array}$ & $0.0523,-0.0156$ \\
\hline 56 & $0.1693,-0.1757$ & $0.0532,0.0230$ & 122 & $\begin{array}{c}0.2492,- \\
0.2470\end{array}$ & $-0.0151,-0.0525$ & 188 & $\begin{array}{c}0.3424,- \\
0.3464\end{array}$ & $0.1074,0.0426$ \\
\hline 58 & $0.1974,-0.2004$ & $0.0390,0.0042$ & 124 & $\begin{array}{c}0.2186,- \\
0.2160\end{array}$ & $-0.0210,-0.0538$ & 190 & $\begin{array}{c}0.3862,- \\
0.3871\end{array}$ & $0.0816,0.0109$ \\
\hline 60 & $0.2174,-0.2180$ & $0.0264,-0.0117$ & 126 & $\begin{array}{c}0.1965,- \\
0.1862\end{array}$ & $-0.0234,-0.0539$ & 192 & $\begin{array}{c}0.3782,- \\
0.3814\end{array}$ & $0.0523,-0.0156$ \\
\hline 62 & $0.2279,-0.2267$ & $0.0149,-0.0249$ & 128 & $\begin{array}{c}0.1216,- \\
0.1455\end{array}$ & $0.1697,0.1406$ & 194 & $\begin{array}{c}0.3664,- \\
0.3668\end{array}$ & $0.0293,-0.0354$ \\
\hline 64 & $0.2270,-0.2246$ & $0.0044,-0.0350$ & 130 & $\begin{array}{c}0.1613,- \\
0.1812\end{array}$ & $0.1298,0.0964$ & 195 & $\begin{array}{c}0.3564,- \\
0.3568\end{array}$ & $0.0253,-0.0334$ \\
\hline
\end{tabular}

Table 4 Magnetic field with vertical displacement by Eqs. (2 and 15)

\begin{tabular}{|c|c|c|c|c|c|c|c|c|}
\hline No. & $B_{x}$ & $B_{z}$ & No. & $B_{x}$ & $B_{z}$ & No. & $B_{x}$ & $B_{z}$ \\
\hline 1 & $\begin{array}{l}-0.0427 \\
-0.0166\end{array}$ & $0.6122,0.1057$ & 66 & $\begin{array}{c}0.5130,- \\
0.5097\end{array}$ & $\begin{array}{c}-0.0093,- \\
0.0820\end{array}$ & 132 & $\begin{array}{c}0.5004,- \\
0.5155\end{array}$ & $0.2098,0.0918$ \\
\hline 3 & $-0.0080-0.0077$ & $0.0657,0.0453$ & 68 & $\begin{array}{c}0.4842,- \\
0.4801\end{array}$ & $\begin{array}{c}-0.0440,- \\
0.0854\end{array}$ & 134 & $\begin{array}{c}0.5353,- \\
0.5446\end{array}$ & $0.0957,0.0826$ \\
\hline 5 & $-0.00038,0.0032$ & $0.0090,-0.0085$ & 70 & $\begin{array}{c}0.4390,- \\
0.4352\end{array}$ & $\begin{array}{c}-0.0518,- \\
0.0855\end{array}$ & 136 & $\begin{array}{c}0.5623,- \\
0.5668\end{array}$ & $0.0954,0.0156$ \\
\hline 7 & $0.0053,0.0050$ & $\begin{array}{c}-0.0421,- \\
0.0418\end{array}$ & 72 & $\begin{array}{l}0.2030,- \\
0.1085\end{array}$ & $\begin{array}{c}-0.0572,- \\
0.0826\end{array}$ & 138 & $\begin{array}{c}0.5810,- \\
0.5819\end{array}$ & $0.0878,-0.0412$ \\
\hline 9 & $0.0095,0.0050$ & $\begin{array}{c}-0.0484,- \\
0.0418\end{array}$ & 74 & $\begin{array}{c}0.0992,- \\
0.0942\end{array}$ & $\begin{array}{c}-0.0574,- \\
0.0782\end{array}$ & 140 & $\begin{array}{c}0.5909,- \\
0.5900\end{array}$ & $0.0523,-0.0585$ \\
\hline 11 & $0.0093,0.0070$ & $\begin{array}{c}-0.0481,- \\
0.0418\end{array}$ & 76 & $\begin{array}{c}0.0092,- \\
0.0809\end{array}$ & $0.5600,0.4928$ & 142 & $\begin{array}{c}0.5903,- \\
0.5890\end{array}$ & $0.0153,-0.0829$ \\
\hline 13 & $0.0085,0.0078$ & $\begin{array}{c}-0.0544,- \\
0.0507\end{array}$ & 78 & $0.0561-0.0872$ & $0.4698,0.4595$ & 144 & $\begin{array}{c}0.5784,- \\
0.5770\end{array}$ & $\begin{array}{c}-0.0142,- \\
0.0828\end{array}$ \\
\hline 15 & 0.0079 & -0.0656 & 80 & $\begin{array}{c}0.0992,- \\
0.2019\end{array}$ & $0.4279,0.4124$ & 146 & $\begin{array}{c}0.5454,- \\
0.5431\end{array}$ & $\begin{array}{c}-0.0462,- \\
0.0885\end{array}$ \\
\hline 16 & $0.0560,-0.0954$ & $0.5298,0.5280$ & 82 & $\begin{array}{c}0.4110,- \\
0.4275\end{array}$ & $0.2090,0.0972$ & 148 & $\begin{array}{c}0.5998,- \\
0.5988\end{array}$ & $\begin{array}{c}-0.0858,- \\
0.0821\end{array}$ \\
\hline 18 & $\begin{array}{c}-0.0855,- \\
0.0627\end{array}$ & $0.4961,0.4345$ & 84 & $\begin{array}{c}0.4504,- \\
0.4618\end{array}$ & $0.0969,0.0892$ & 150 & $\begin{array}{c}0.4986,- \\
0.5179\end{array}$ & $0.4501,0.4072$ \\
\hline 20 & $0.0157,-0.0740$ & $0.4398,0.4383$ & 86 & $\begin{array}{l}0.4851,- \\
0.4918\end{array}$ & $0.0887,0.0556$ & 152 & $\begin{array}{c}0.5340,- \\
0.5513\end{array}$ & $0.4147,0.0989$ \\
\hline 22 & $0.0824,-0.0962$ & $0.4062,0.4059$ & 88 & $\begin{array}{c}0.5131,- \\
0.5162\end{array}$ & $0.0830,0.0098$ & 154 & $\begin{array}{c}0.5668,- \\
0.5803\end{array}$ & $0.1066,0.0665$ \\
\hline 24 & $0.1072,-0.4010$ & $0.1030,0.1030$ & 90 & $\begin{array}{l}0.5328,- \\
0.5334\end{array}$ & $0.0693,-0.0415$ & 156 & $\begin{array}{c}0.0636,- \\
0.6018\end{array}$ & $0.0932,0.0400$ \\
\hline 26 & $0.3257,-0.4353$ & $0.0949,0.0949$ & 92 & $\begin{array}{c}0.5431,- \\
0.5420\end{array}$ & $0.0469,-0.0559$ & 158 & $\begin{array}{c}0.6143,- \\
0.6156\end{array}$ & $0.0833,-0.0426$ \\
\hline 28 & $0.3599,-0.4660$ & $0.0897,0.0898$ & 94 & $\begin{array}{c}0.5421,- \\
0.5400\end{array}$ & $0.0094,-0.0766$ & 160 & $\begin{array}{c}0.6242,- \\
0.6237\end{array}$ & $0.0557,-0.0613$ \\
\hline 30 & $0.4879,-0.4910$ & $0.0765,0.0765$ & 96 & $\begin{array}{c}0.5290,- \\
0.5243\end{array}$ & $\begin{array}{c}-0.0462,- \\
0.0853\end{array}$ & 162 & $\begin{array}{c}0.6249,- \\
0.6245\end{array}$ & $0.0400,-0.0765$ \\
\hline 32 & $0.4081,-0.5089$ & $0.0545,0.0546$ & 98 & $\begin{array}{c}0.5140,- \\
0.5820\end{array}$ & $\begin{array}{c}-0.4809,- \\
0.5115\end{array}$ & 164 & $\begin{array}{c}0.5914,- \\
0.5890\end{array}$ & $\begin{array}{c}-0.0132,- \\
0.0848\end{array}$ \\
\hline 34 & $0.5186,-0.5175$ & $0.0437,0.0437$ & 100 & $\begin{array}{c}0.4653,- \\
0.5391\end{array}$ & $\begin{array}{c}-0.4531,- \\
0.4770\end{array}$ & 166 & $\begin{array}{c}0.6086,- \\
0.6078\end{array}$ & $\begin{array}{c}-0.0090,- \\
0.0864\end{array}$ \\
\hline
\end{tabular}


Table 4 continued

\begin{tabular}{|c|c|c|c|c|c|c|c|c|}
\hline No. & $B_{x}$ & $B_{z}$ & No. & $B_{x}$ & $B_{z}$ & No. & $B_{x}$ & $B_{z}$ \\
\hline 36 & $0.5179,-0.5153$ & $0.0087,0.0079$ & 102 & $\begin{array}{c}0.0987,- \\
0.1000\end{array}$ & $0.4958,0.4798$ & 168 & $\begin{array}{c}0.5806,- \\
0.5920\end{array}$ & $0.4320,0.0958$ \\
\hline 38 & $0.5042,-0.5006$ & $\begin{array}{c}-0.0114,- \\
0.0103\end{array}$ & 104 & $\begin{array}{c}0.2007,- \\
0.4153\end{array}$ & $0.4464,0.4253$ & 170 & $\begin{array}{c}0.6125,- \\
0.6219\end{array}$ & $0.2094,0.0803$ \\
\hline 40 & $0.4761,-0.4717$ & $\begin{array}{c}-0.0516,- \\
0.0435\end{array}$ & 106 & $\begin{array}{c}0.4326,- \\
0.4512\end{array}$ & $0.4127,0.1061$ & 172 & $\begin{array}{c}0.6380,- \\
0.6435\end{array}$ & $0.0924,0.0407$ \\
\hline 42 & $0.4321,-0.4274$ & $\begin{array}{c}-0.0516,- \\
0.0515\end{array}$ & 108 & $\begin{array}{c}0.4722,- \\
0.4851\end{array}$ & $0.1070,0.0849$ & 174 & $\begin{array}{c}0.6570,- \\
0.6583\end{array}$ & $0.0894,-0.0445$ \\
\hline 44 & $0.1051,-0.1004$ & $\begin{array}{c}-0.0561,- \\
0.0557\end{array}$ & 110 & $\begin{array}{c}0.4574,- \\
0.4542\end{array}$ & $0.0963,0.0595$ & 176 & $\begin{array}{c}0.6664,- \\
0.6666\end{array}$ & $0.0593,-0.0654$ \\
\hline 46 & $\begin{array}{c}-0.0127,- \\
0.0598\end{array}$ & $0.5131,0.5102$ & 112 & $\begin{array}{c}0.5071,- \\
0.5146\end{array}$ & $0.0886,0.0121$ & 178 & $\begin{array}{c}0.6662,- \\
0.6666\end{array}$ & $0.0426,-0.0818$ \\
\hline 48 & $0.0421,-0.0800$ & $0.4510,0.4449$ & 114 & $\begin{array}{c}0.5348,- \\
0.5385\end{array}$ & $0.0632,-0.0415$ & 180 & $\begin{array}{c}0.6363,- \\
0.6362\end{array}$ & $0.0402,-0.0886$ \\
\hline 50 & $0.0855,-0.0953$ & $0.4144,0.4023$ & 116 & $\begin{array}{c}0.5541,- \\
0.5547\end{array}$ & $0.0494,-0.0567$ & 182 & $\begin{array}{c}0.6424,- \\
0.6465\end{array}$ & $0.4074,0.0825$ \\
\hline 52 & $0.1059,-0.4111$ & $0.1090,0.0906$ & 118 & $\begin{array}{c}0.5641,- \\
0.5632\end{array}$ & $0.0109,-0.0690$ & 184 & $\begin{array}{c}0.6862,- \\
0.6871\end{array}$ & $0.1016,0.0409$ \\
\hline 54 & $0.4549,-0.4453$ & $0.0994,0.0848$ & 120 & $\begin{array}{c}0.5635,- \\
0.5618\end{array}$ & $\begin{array}{c}-0.0117,- \\
0.0878\end{array}$ & 186 & $\begin{array}{c}0.6782,- \\
0.6816\end{array}$ & $0.0823,-0.0457$ \\
\hline 56 & $0.4893,-0.4757$ & $0.0852,0.0730$ & 122 & $\begin{array}{c}0.5492,- \\
0.5470\end{array}$ & $\begin{array}{c}-0.0451,- \\
0.0825\end{array}$ & 188 & $\begin{array}{c}0.6424,- \\
0.6464\end{array}$ & $0.4074,0.0826$ \\
\hline 58 & $0.4574,-0.5104$ & $0.0720,0.0653$ & 124 & $\begin{array}{c}0.5186,- \\
0.5160\end{array}$ & $\begin{array}{c}-0.0510,- \\
0.0838\end{array}$ & 190 & $\begin{array}{c}0.6862,- \\
0.6871\end{array}$ & $0.1016,0.0400$ \\
\hline 60 & $0.5674,-0.5380$ & $0.0614,-0.0547$ & 126 & $\begin{array}{c}0.4965,- \\
0.4862\end{array}$ & $\begin{array}{c}-0.0534,- \\
0.0839\end{array}$ & 192 & $\begin{array}{c}0.6782,- \\
0.6814\end{array}$ & $0.0823,-0.0456$ \\
\hline 62 & $0.5479,-0.5567$ & $0.0429,-0.0409$ & 128 & $\begin{array}{c}0.4216,- \\
0.4455\end{array}$ & $0.4697,0.4406$ & 194 & $\begin{array}{c}0.6664,- \\
0.6668\end{array}$ & $0.0593,-0.0653$ \\
\hline 64 & $0.5370,-0.5546$ & $0.0083,-0.0590$ & 130 & $\begin{array}{c}0.4613,- \\
0.4812\end{array}$ & $0.4298,0.2064$ & 195 & $\begin{array}{c}0.6564,- \\
0.6568\end{array}$ & $0.0553,-0.0665$ \\
\hline
\end{tabular}

loads that appeared. Consequently, halo current fraction and toroidal peaking factor (TPF) in vessel components depend upon the halo current density. In MHD simulation problems, plasma model comprises of three regions, namely, core, halo, and resistive wall region integrating plasma to external vacuum magnetic field. In EAST reactor, plasma was inherently unstable against vertical displacement and during upward and downward movement creates disruption along with large halo current generation. In this case, when the plasma flows into the vacuum vessel through in-vessel components, halo current produced large values of $J \times B$ forces acting on the vessel through invessel components. The production and movement of halo current is such that it first appeared on the outer plate in clockwise direction and maximum generation of halo current was estimated to be about 0.4 times of the plasma current. Figure 3 shows the evolution of halo current and filament. EAST halo current is $10 \mathrm{KA}$ for one cassette and total 400KA recorded by model (18). In EAST, Rogowski coils have been designed for both the upper and lower diverters to measure the disruption of halo currents. EAST upper diverter was upgraded with a new tungsten diverter consisting of 80 cassettes in the toroidal direction. Four upper diverter, cassettes have been instrumented with a set

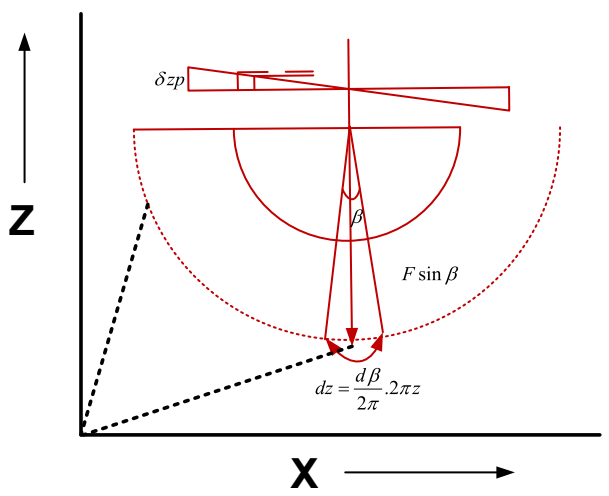

Fig. 2 Plasma orientation (case 2) 


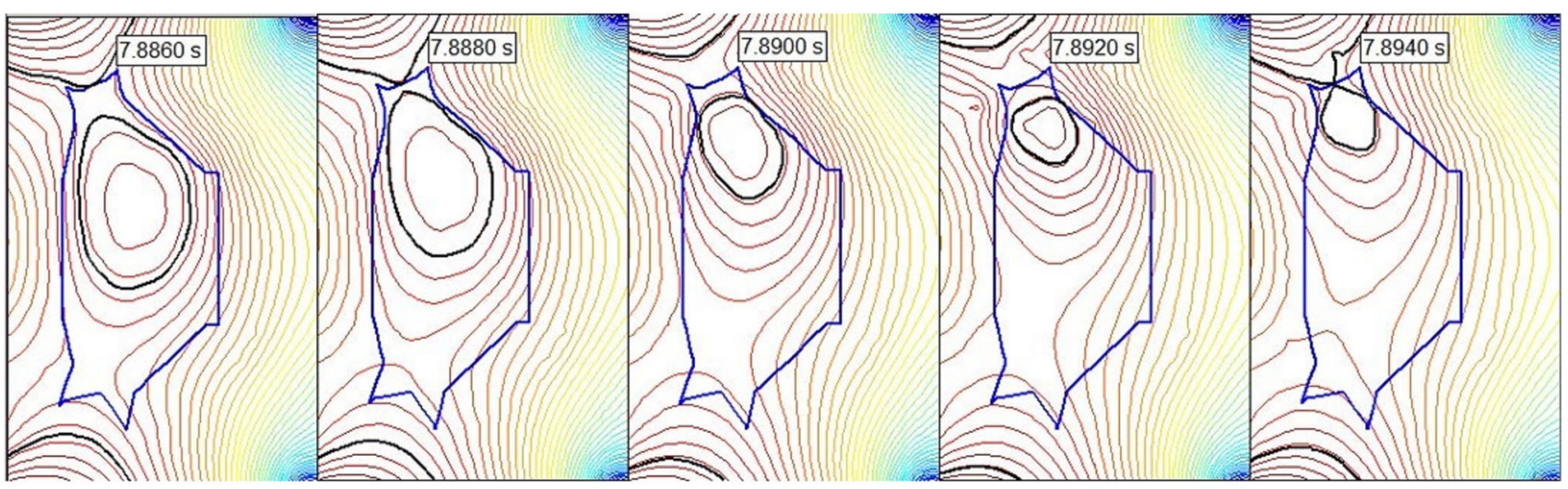

(a)
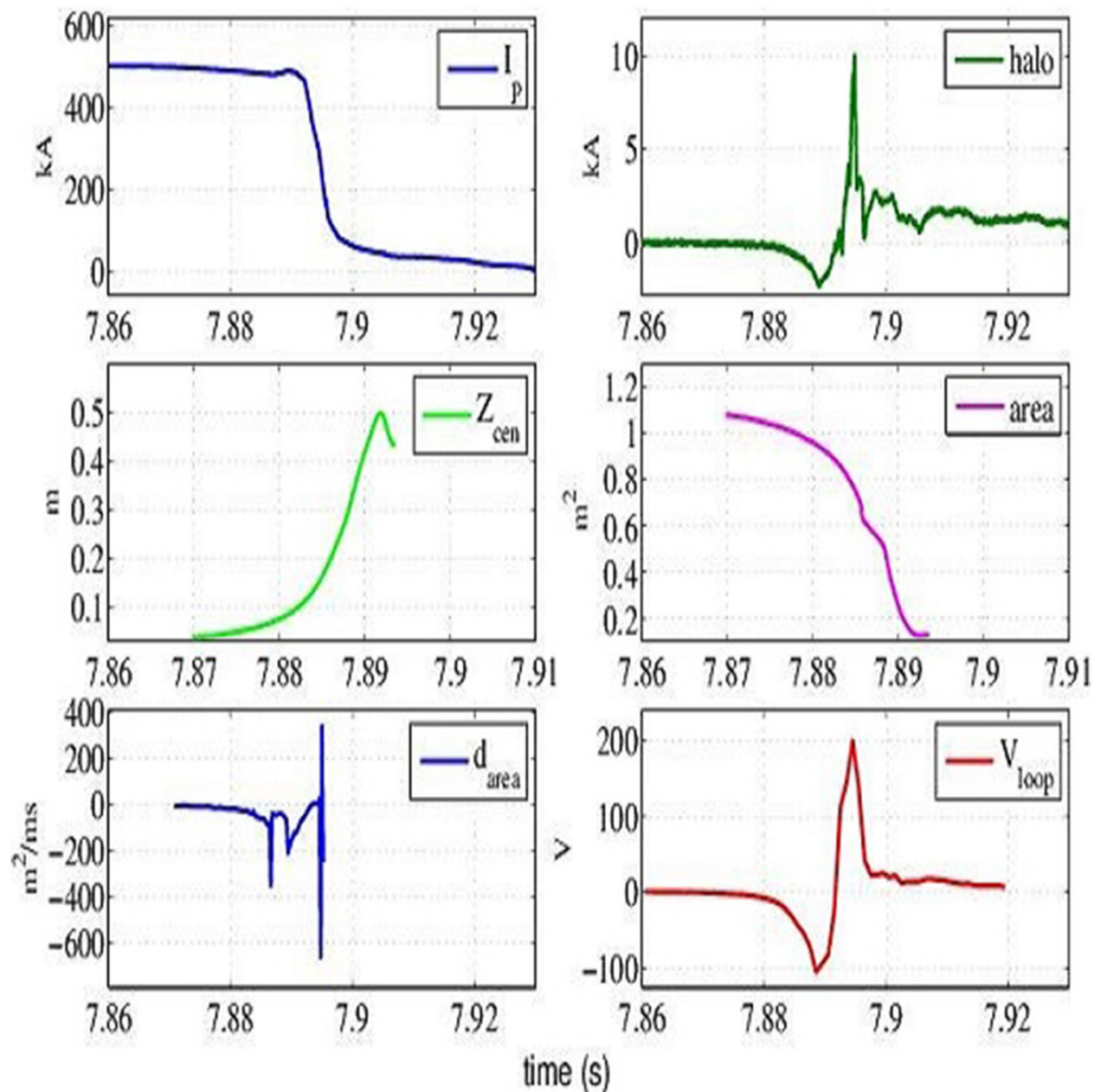

(b)

Fig. 3 Evolution of halo current and filament

of 10 small-cross sections. Rogowski coils to determine where the halo currents enter and exit the diverter, and how much current flows through the water cooling tubes. In this paper, we have successfully performed theoretical investigation between halo current-plasma deformation/ displacement and theoretical calculation of total halo currents as well. 


\section{Conclusion}

The developed theoretical model calculates plasma cross sections by $B_{x}$ and $B_{z}$ magnetic field points and displacement subjected to the start of VDE and magnetic field flux variations. The developed two conducting points give an indication to halo current percentages as well. This model can calculate theoretically halo current during the disruption phases in a very short time. Furthermore, mathematical techniques have been developed successfully which shows the relation between halo currents and plasma displacement/deformation in EAST Tokamak. Computational program has been developed to calculate total halo current and magnetic field calculation points. Theoretical investigation of each cassette has been calculated by model. This model can be subjected to experimental data for other Tokamak devices as well.

Acknowledgements The authors would like to sincerely appreciate the Deanship of Scientific Research at King Saud University for its funding of this research through the Research Group Project No. RGP-255.

Open Access This article is distributed under the terms of the Creative Commons Attribution 4.0 International License (http://creative commons.org/licenses/by/4.0/), which permits unrestricted use, distribution, and reproduction in any medium, provided you give appropriate credit to the original author(s) and the source, provide a link to the Creative Commons license, and indicate if changes were made.

\section{References}

1. Riccardo, V., et al.: Progress in understanding halo current as JET. Nucl. Fusion 49, 8 (2012)

2. ITER Physics Expert Group on Disruptions: Plasma Control, MHD and ITER Physics Basis Editors. Nucl. Fusion 49, 8 (1999)

3. Riccardo, V., Walkerb, S., Nollc, P.: Modelling magnetic forces during asymmetric vertical displacement events in JET. Fusion Eng. Des. 47(4), 389-402 (2000)

4. Neyatani, Y., Yoshino, R., Nakamura, Y., Sakurai, S.: Characteristics of halo currents in JT-60U. Nucl. Fusion 39(4), 559 (1999)

5. Gerhardt, S.P.: Dynamics of the disruption halo current toroidal asymmetry in NSTX. Nucl. Fusion 53, 023005 (2013)

6. Normile, D.: Waiting for ITER. Fusion jocks look EAST. Science. 312, 992-993 (2006)

7. Wan, B.N.: Recent experiments in the EAST and HT-7 superconducting Tokamaks. Nucl. Fusion 49, 104011 (2009)

8. Li, J., Wan, B.N.: Recent progress in RF heating and long-pulse experiments on EAST. Nucl. Fusion 51, 094007 (2011)

9. Wang, X., et al.: Progress of high power and long pulse ECRH system in EAST. Fusion Eng. Des. 96-97, 181-186 (2015)
10. Wan, B., for the EAST andHT-7 Teams and International Collaborators: Recent experiments in the EAST and HT-7 superconducting tokamaks. Nucl. Fusion 49, 104011 (2009)

11. Wan, Y.X., Li, L., Weng, P., and EAST team.: Overview progress and future plan of the east project. In: OV/1-1 on 21th IAEA Fusion Energy Conference. Chengdu. China. (2006)

12. Li, J., et al.: A long-pulse high-confinement plasma regime in the Experimental Advanced Superconducting Tokamak. Nat. Phys. 9. 817. 2013

13. Li, J., et al.: Plasma facing components for the Experimental Advanced Superconducting Tokamak and CFETR. Phys. Scr. T159, 014001 (2014)

14. Granetz, R.S., et al.: 7th US-PRC Magnetic Fusion Collaboration Workshop. 28. http://www.swip.ac.cn/mfcw2014/7th\%20USPRC\%20MFC\%20abstracts.pdf\#page $=29.2014$

15. Long, C.D., et al.: Characterization of plasma current quench during disruption in EAST tokamak. Chin. Phys. B 24, 025205 (2015)

16. Strauss, H.R. et al.: Halo current and resistive wall simulations of ITER. 1-7.TH/2-2

17. Long, C.D. et al.: Observation and analysis of halo current in EAST. Chin Phys B. 23, 065205 (2014)

18. Strauss, H.R.: Non-linear MHD halo current simulations, TH/2-3

19. Fabtechi, S., Crutzen, Y.: Modelling and analysis of plasma-first wall contact during vertical instabilities in next-step tokamaks with a 3D eddy current code. In: Fusion Technology 1996. Proceedings of the 19th Symposium on Fusion Technology, Lisbon, Portugal, 16-20 September 1996, pp. 727-730. Elsevier, Amsterdam (1997)

20. Long, C.D., et al.: Halo current diagnostic system of experimental advanced superconducting tokamak. Rev. Sci. Instrum. 86, 103506 (2015)

21. Pironti, A., et al.: Optimal choice of the geometrical descriptors for tokamak plasma shape control. Fusion Eng. Des. 43, 115-127 (1998)

22. Kurihara, K.: A new shape reproduction method based on the Cauchy-condition surface for real-time Tokamak reactor control. Fusion Eng. Des. 51-52, 1049-1057 (2000)

23. Hofinann, F., et al.: Equilibrium and axisymmetric stability of the proposed TCV Tokamak. 14th Symposium on Fusion Technology. Fusion Technol. 1, 687-692 (1986)

24. Wesson, J.: Tokamaks. Oxford Univ Press, Oxford (2011)

25. Yuntao, S., Wu, W., Du, S.: Tokamak engineering mechanics. Mechanical Engineering Series. Springer, Berlin (2014). ISBN: 978-3-642-39574-1

26. Bachmann, C.: Asymmetric forces on the ITER plasma in kink mode in subsequent halo currents in the VV. ITER_D_28P25D v1.0. (2015)

27. Forces on the ITER Vacuum Vessel due to the Plasma in Kink Mode during a VDEIII with Slow Current Quench, ITER_D_26RLC9

\section{Publisher's Note}

Springer Nature remains neutral with regard to jurisdictional claims in published maps and institutional affiliations. 